\title{
The Influence of Solvents on the Stability of Carbonated Hydroxyapatite ${ }^{\dagger}$
}

\author{
Diana Alexandra Minea ${ }^{1}$, Rodica-Mariana Ion 1,2 and Ana-Alexandra Sorescu 1,2,* \\ 1 National Institute for Research \& Development in Chemistry and Petrochemistry - ICECHIM Bucharest, \\ 202 Spl. Independentei, 060021 Bucharest, Romania; dee.minea07@gmail.com (D.A.M.); \\ rodica_ion2000@yahoo.co.uk (R.-M.I.) \\ 2 Valahia University, Materials Engineering Department, 130004 Targoviste, Romania \\ * Correspondence: ana-alexandra.sorescu@icechim.ro or alexiasorescu@yahoo.com \\ + Presented at the 15th International Symposium "Priorities of Chemistry for a Sustainable Development" \\ PRIOCHEM, Bucharest, Romania, 30th October-1st November 2019.
}

Published: 15 October 2019

Keywords: hydroxyapatite; solvents; stability of consolidants

Hydroxyapatite is an inorganic consolidant used in the restoration of cultural heritage. For this reason, the study of the effects of different agents on hydroxyapatite is paramount [1,2]. In this paper, the effect of solvents (isopropyl and tert-Butyl alcohol) was investigated by measuring the stability of absorbance over time.

To this end, solutions containing water and alcohol in different proportions were prepared, starting from the binary solutions containing water: Alcohol $(0-100 \%)$ with a constant mass of hydroxyapatite added, and the absorbance was measured over a period of time at a fixed wavelength, $\lambda=600 \mathrm{~nm}$.

For this study, a JK-VS-721N visible spectrophotometer was used to measure the absorbance of 11 solutions containing water $(0-100 \%)$ and isopropyl alcohol or tert-Butyl alcohol $(0-100 \%)$ and hydroxyapatite.

For a hydroxyapatite suspension to be effective as a consolidant, the sedimentation rate of this solution should be as low as possible so that the respective suspension is homogeneous when applied to a stone surface [3]. In our case, with hydroxyapatite in the binary water mixture: Alcohol (60-40\%) has the lowest sedimentation rate, thus being the most efficient solution for applying on the stone surface (Figure 1).
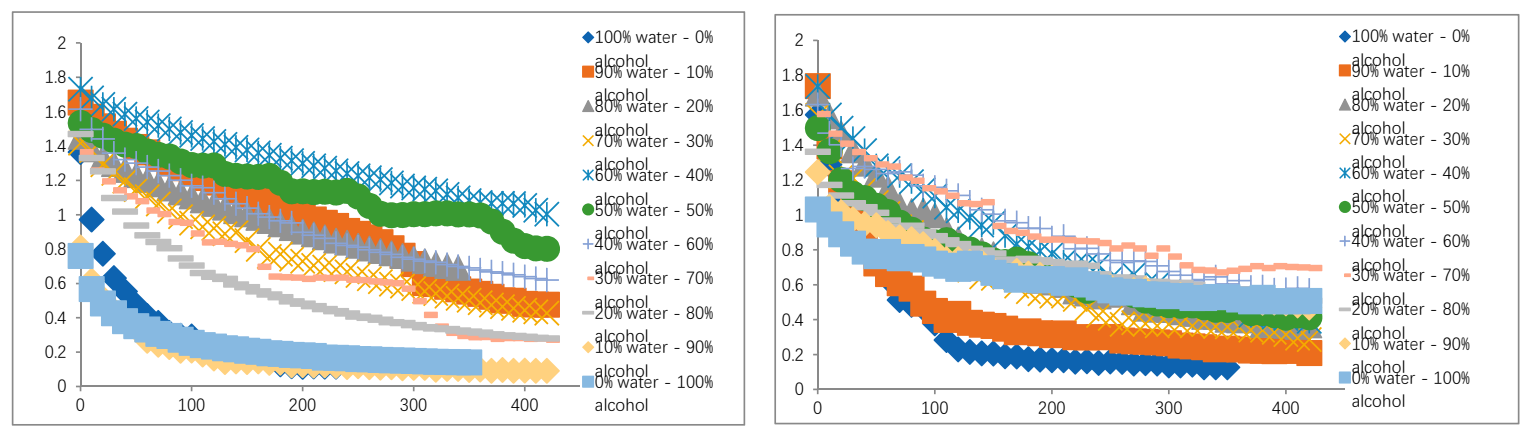

Figure 1. Absorbance variation in time for solutions containing water/isopropyl alcohol (left) and water/tert-Butyl alcohol (right). 
In the case of hydroxyapatite in the binary water mixture: Alcohol $(60-40 \%)$ has the lowest sedimentation rate, being the most efficient solution for application on the stone surface.

Acknowledgments: This work was supported by a grant of the Romanian Ministry of Research and Innovation, CCCDI-UEFISCDI, project number PN-III-P1-1.2-PCCDI-2017-0476/51PCCDI/2018, within PNCDI III, PN 19.23.03.01.04, and 5PS/2019.

\section{References}

1. Guo, X.; Xiao, P. Effects of solvents on properties of nanocrystalline hydroxyapatite produced from hydrothermal process. J. Eur. Ceram. Soc. 2006, 26, 3383-3391.

2. Sassoni, E.; Graziani, G.; Franzoni, E.; Scherer, G. W. Calcium phosphate coatings for marble conservation: Influence of ethanol and isopropanol addition to the precipitation medium on the coating microstructure and performance. Corros. Sci. 2018, 136, 255-267.

3. Sassoni, E.; Naidu, S.; Scherer, G. W. The use of hydroxyapatite as a new inorganic consolidant for damaged carbonate stones. J. Cult. Herit. 2011, 12, 346-355.

C 2019 by the authors. Licensee MDPI, Basel, Switzerland. This article is an open access article distributed under the terms and conditions of the Creative Commons Attribution (CC BY) license (http://creativecommons.org/licenses/by/4.0/). 